\title{
Revisiting the mechanism behind the similar size of slums
}

\author{
John Friesen, ${ }^{*}$ Jakob Hartig, and Peter F. Pelz \\ Chair of Fluid Systems \\ Technische Universität Darmstadt
}

(Dated: December 22, 2020)

\begin{abstract}
Worldwide, about one in eight people live in slums. Empirical studies based on satellite data have identified that the size distributions of this type of settlement are similar in different cities of the Global South. Based on these results, we developed a model describing the formation of slums with a Turing mechanism, in which patterns are created by diffusion-driven instability and the inherent characteristic length of the system is independent of boundary conditions. We examine the model in this paper by critically reflecting its assumptions, comparing them with recent empirical observations and discussing possible adjustments and future extensions based on new methods of identifying pattern formation mechanisms.
\end{abstract}

\section{INTRODUCTION}

Worldwide, about 1 billion people currently live in socalled slums or informal settlements. Settlement structures or households are defined by the United Nations as slums if they meet at least one of the following five characteristics: no security of tenure, no access to adequate sanitaiton, no easy access to safe water, no sufficient living space or non-durable housing [1]. Especially the latter characteristic is used in recent studies to distinguish settlements of urban poverty from other urban settlement structures by means of satellite images [2-4]. Since the methodology used in these studies can only capture the physical structure or morphology of this type of settlement, they are also referred to as morphological slums. This term is used to describe settlement forms that differ from their surroundings in their high density, low building heights and organic, complex structures. Although recent studies have shown that this form of settlement correlates with a special social group of low income and with urban poverty [5], there has been intensive discussion in recent years about an appropriate language and terminology, which on the one hand perceives the specific living conditions of the inhabitants of these settlements and their reality of life, but at the same time does not stigmatize them by using negatively connotated terms (cf. [6-8] for general considerations on the terminology and [9-11] for discussions in the context of concrete studies). Since in the following we examine in more detail a model for the emergence of these structures, we refer to the physical settlement structures as morphological slums and its inhabitants as morphological slum dwellers. We do this in the awareness that the realities of the inhabitants' lives can differ greatly. The term slum is nevertheless included the term morphological slum used by us, in relation to the many studies on slums and informal settlements that primarily examine the effects of urban inequality and deprivation.

The main aim of studies on morphological slums is to

\footnotetext{
* john.friesen@fst.tu-darmstadt.de
}

reduce the currently still large gaps in knowledge about this type of settlement [12] and to be able to compare the different regional characteristics and their spatial characteristics with globally uniform methods. One step in this direction was done by Friesen et al. [13, 14] who analyse the size distribution of morphological slums in eight different cities in the Global South. They find that morphological slums show similar size distributions with a similar geometric mean. This leads to the conclusion that regardless of culture, country or continent, the size of most slums (nearly $85 \%$ ) is between $10^{-3}$ and $10^{-1}$ $\mathrm{km}^{2}$ and thus shows no dependence on the total number of morphological slums within the city. This information can be used as typical scale in other scientific domains such as infrastructural planning [15] or epidemiological analyses [16, 17].

These studies on the similar size of morphological slums were recently taken up by Pelz et al. [18] who put forward the hypothesis that the development of morphological slums could be described by a Turing mechanism [19]. This is inspired by the fact, that a characteristic scale can be observed in both Turing patterns and morphological slums. The emergence of morphological slums is understood as a process of self-organization, in which morphological slums are the result of the interaction of two social groups. This hypothesis was not sufficiently discussed in its presentation [18]. We are also not aware of any other work that has critically examined the assumptions and consequences of the model.

Hence, in this paper, we discuss the model [18] in the context of current research results on different levels and elaborate possible future perspectives. To this end, we first present the original model and its basic assumptions and conclusions (sec. II). In a further step, we discuss in detail the

- model assumptions and ethnographic labels (sec. III),

- the spatial and temporal properties of the empirical data and the model (sec. IV) and

- possible model extensions and the possibility of parameter identification with regard to current re- 
search (sec. V).

After some general reflections on the model (sec. VI), we finally conclude (sec. VII) this communication.

\section{MODEL}

In order to understand the development of morphological slums, different models have been presented in the literature [20], which often use a large number of parameters to depict the different socio-economic processes leading to the formation of morphological slums, especially in cities of the Global South. In contrast, Pelz et al. [18] presented a model in which the formation of morphological slums results from the interaction of two social groups.

The population is divided into two groups, "rich" and "poor", on the basis of income and the behaviour and interaction of both groups is described by two coupled partial differential equations

$$
\begin{aligned}
\frac{\partial \widetilde{u}_{1}}{\partial \widetilde{t}} & =\widehat{U} R f_{1}\left(u_{1}, u_{2}\right)+D_{1} \Delta \widetilde{u}_{1}, \\
\frac{\partial \widetilde{u}_{2}}{\partial \widetilde{t}} & =\widehat{U} R f_{2}\left(u_{1}, u_{2}\right)+D_{2} \Delta \widetilde{u}_{2},
\end{aligned}
$$

where $\widetilde{u}_{i}$ describes the density fields of both population groups ( $i=1$ represent "rich", $i=2$ represent "poor"), $\widehat{U}$ the population density and $D_{i}$ the diffusion coefficients. $R$ has the dimenson of a rate and $f_{i}$ are the coupled reaction kinetics.

These equations describe the change of the concentration of inhabitants as the sum of dwellers moving into, being born or dying within the city (reaction term) and moving within the city (diffusion term).

The equations are brought into a dimensionless form by using the following parameters $t:=R \widetilde{t}, x_{j}:=\widetilde{x}_{j} \sqrt{R / D_{1}}$, $u_{i}:=\widetilde{u}_{i} / \widehat{U}$ leading to

$$
\frac{\partial u_{i}}{\partial t}=f_{i}\left(u_{j}\right)+d_{i j} \frac{\partial^{2} u_{j}}{\partial x_{k} \partial x_{k}},\left(d_{i j}\right)=\left(\begin{array}{ll}
1 & 0 \\
0 & d
\end{array}\right) .
$$

Thus, instead of describing the behavior of the different inhabitants of the city separately (as is done, for example, in agent-based models [20]), their behavior is averaged and described by means of partial differential equations. The authors interprete the behavior of the two groups by using two basic phenomena: short distance migration and long distance migration.

While the short distance migration, which is represented by the diffusion term, depends only on the concentration of the respective population group at a specific location, the interaction of the two groups is represented in the coupled reaction terms, called long distance migration.

The basic idea of the model is that a stable state of long-distance migration is assumed, in which the interaction between the two social groups leads to an even distribution. This corresponds to the analogy from the model of Turing, where the system is stable in the absence of diffusion [19]. This initial stable state can be described by certain conditions, which are expressed in a special form of the Jacobian $a_{i j}:=\frac{\partial f_{i}}{\partial u_{j}}$, called behavior matrix in the model. For detailed derivations and necessary as well as sufficient conditions for the formation of patterns please refer to the standard literature, e.g. [21].

A necessary condition for a stable state is a negative trace and a positive determinant of the Jacobian . To fulfill this conditions, the Jacobian has to have one of the following four proporties

$$
\left(\begin{array}{ll}
+ & + \\
- & -
\end{array}\right),\left(\begin{array}{ll}
- & - \\
+ & +
\end{array}\right),\left(\begin{array}{ll}
+ & - \\
+ & -
\end{array}\right),\left(\begin{array}{ll}
- & + \\
- & +
\end{array}\right) .
$$

While the first two Jacobians represent activatorsubstrate models, the latter ones are activator-inhibitor models [22]. In the model of Pelz et al. [18] the self organization of morphological slums is interpreted as activator-substrate model, since in the model it is assumed that the courses of the population density are in opposite phases, i.e. that in places where many inhabitants of formal settlements live, few morphological slum dwellers live, and vice versa.

However, due to different migration behaviour of the two groups, described by the diffusion terms in the equation, the system can be transformed from a stable to an unstable state in which the faster diffusion or mobility of the more wealthier population group leads to a regular pattern formation (cf. Figure 3 (iv)). The principle of the Turing mechanism can be understood by looking at Figure 2, A. In a self-amplification reaction, the activator multiplies as the substrate is consumed. Since the substrate is produced at any point in the system and diffuses faster than the activator, patterns are formed [22, 23]. In order to generate binary patterns as shown in Figure 1 on the basis of continuous concentration curves, a threshold value is introduced. If the concentration of the activator in a certain location is above a threshold, this location is assigned the value 1 , if the concentration is below, the value 0 . In this way a binary pattern is generated from a continuous concentration distribution. Detailed descriptions of how binary patterns, such as on animal coats, are generated from such concentration distributions can be found in [21]. How this threshold is defined remains unmentioned in [18].

While other models usually take a people-centred view, the model of Pelz et al. [18] is able to depict an empirically observed quantity, independent, of the city, country and culture the morphological slums are found in. In the model, this is the result of the simple interaction of two groups, whose behaviour can be represented by partial differential equations and thus analytically investigated.

Like every model, the presented model [18] is based on a number of assumptions, which we examine in more detail below. In a further step, we compare these assumptions in detail with current results from empirical 
research on morphological slums and with possible approaches to extend the model.

\section{MODEL ASSUMPTIONS}

\section{A. Terminology and ethnographic labels}

We first start by analysing the terminology used in the model. The classification of rich and poor is used in various studies to designate population groups (e.g. [24]). Already Plato speaks of the fact that the city can be divided into a rich and a poor city [25, 26]. This distinction is made in many further studies, sometimes however without a clear definition of the threshold (cf. $[27])$ and also in the presented model and needs a critical discussion.

In the literature it is repeatedly mentioned that simply dichotomous classifications can be problematic and perceived as judgmental, because people, groups of people or whole states are often reduced to a single characteristic and categorized according to that characteristic [28].

The question arises how to distinguish between the two population groups. That is, from which border on the population is classified as poor, from which as rich. This discussion is well known in poverty research, and leads to distinctions between relative and absolute poverty [29]. While relative poverty affects those parts of the population whose wealth is in a certain lower percentile of the wealth distribution, absolute poverty is defined by the fact that the respective person or group has less than a certain amount of money available per day.

In the model [18] the population is divided into two groups based on the respective income of the individuals. Since only two groups were studied, the model implies that the behavioural characteristics described in the behaviour matrix change due to monetary aspects. This means that if the whole population is divided into two groups and the wealth distribution in the city under consideration is continuous, the behaviour of a specific person close to the poverty level would change in dependence on the poverty threshold, what would be a strong assumption.

Furthermore, the model describes the formation of morphological slums as a process in which the members of a mixed population consisting of two groups interact with each other. It draws on an analogy from chemistry or biology, in which the first empirical evidence for pattern formation by the Turing mechanism was presented [21]. While in the chemical studies of pattern formation the morphogens are dissolved in a liquid and can thus interact with each other [30], it remain unknown what the analogy to such a liquid in the described model is. Also, self-reinforcing effects in an activator-substrate model assume an interaction between both morphogens, which then produce new activator morphogens. However, it is difficult to derive an analogy to this behavior in the proposed model, since the formal dwellers would be the substrate for the morphological slum dwellers.

Lastly, it should be mentioned that easy allocation to two-part groups in terms of income is often difficult, as even small increases in income can have a significant impact on a person's circumstances, which is why categorisations often have more than two classes [31]. The behaviour and interaction of different groups could be integrated into the model, but this would increase the complexity and raise questions about the advantages of the model over other approaches, such as agent-based models. Another way to deal with these challenges in the model would be a more abstract designation of morphogens, as postulated by Levashova et al. [32], for example. However, this would only shift the underlying problem of defining morphogens to another level.

\section{B. Initial and boundary conditions}

The model implicitly states the assumption, that the process of pattern formation starts from a homogeneous area. This is a simplification of the real conditions, because groups of people build dwellings for shelter, even if they are only temporary [3]. Even simple settlement structures like tents, are a physical barrier to the movement of the population groups and can affect the mobility leading to an anisotropic behaviour. Particularly in the Global South, the phenomenon of gated communities is a recurring phenomenon [33], in which more affluent population groups shield themselves from the rest of society, which can be interpreted as strong barriers to diffusion. This calls into question the assumption of isotropic diffusion stated in the model.

Neumann boundary conditions with zero-flux were chosen. This assumption is based on the fact that the population traveling into the city can be mapped in the reaction term via long-distance migration, while within the city only short-distance migration takes place.

Transfering the model on a real city, the geographical limitations and barriers of the system (such as mountains or the sea) lead to fact that only certain wavelengths can be formed [21]. If the wavelengths caused by the Turing instability are greater than the characteristic length of the investigated area, no patterns can form despite the necessary conditions for pattern formation derived by a stability analysis being fulfilled. Furthermore, it has to be considered that a change of the area size leads to a change of the wavelength forming in the system [21].

The points mentioned in this section highlight the tension between the concept, presented by Pelz et al. [18] and a application of the model to a real system, which should be addressed in further studies of the model.

\section{Modelling of human behaviour}

Another central question in the discussion of the model presented is the representation of human movement and 
the connected decision making. The assumption is made in many models that human movement can be represented by a random walk and that the movement of a large number of people can thus be represented by diffusion coefficients. An extensive review of random walk models can be found in [34].

Gonzalez et al. [35] investigate the movement behaviour of people on the basis of mobile phone data and conclude that the movement follows patterns with special spatial and linear characteristics. They show that movements of the people follow a random walk, with a the step size following a power law. [36]. Song et al. [37] further show that the mobility behavior of people follows regularities that can be predicted with a high probability. They also investigate the limits of this predictability in detail [37]. These limits have been investigated in more detail in further work [38].

With regard to the model presented [18] it must be stated that the assumption that human behaviour can be modelled by means of random walk is possible, but that the approaches to the mobility of the different groups described there should be refined.

\section{SPATIAL AND TEMPORAL PROPERTIES}

In addition to the fundamental validity of the assumptions discussed above, it must also be examined whether the spatial arrangement and temporal development of morphological slums show similarities to Turing patterns.

\section{A. Spatial and size distribution of morphological slums and concentration peaks}

Comparing the pattern formation of morphological slums Figure 1, B with the Turing patterns in Figure 1, A, clear differences are already noticeable visually. Besides shape and size, the morphological slums show an arrangement in which certain areas of the city are not covered with morphological slums. In previous studies it was shown that slums are arranged in clusters [39]. Hartig et al. [40] confirmed and extended these findings by showing a tendency towards random spatially distribution of morphological slums within the clusters. Furthermore, it can be seen that morphological slums show a wider size distribution than the patterns in the RD equations.

The above-mentioned studies have shown that the size distributions of morphological slums in different cities in the Global South are similar and have almost identical geometric means $[13,14]$. However, while these size distributions show a wide variance (Figure 1, B), size distributions of concentration peaks of Turing patterns are usually very narrow (Figure 1, A).

\section{B. Temporal development}

If the question is investigated whether morphological slums are Turing patterns, not only the spatial distribution of the resulting pattern should show the characteristics of a Turing system, but also its temporal development, as Kondo and Miura [43] have shown.

The formation of Turing patterns in an RD system is based on the interaction of two morphogens, whose temporal development of the concentration curves are interrelated, as can be seen in Figure 2, A. Due to the coupling in the differential equations, an increase in the activator concentration is automatically associated with a decrease in the substrate concentration.

This behaviour is not generally present in the formation of morphological slums. Often morphological slums are built on open or green spaces within the city, [3], removed by external circumstances, such as flooding or politically motivated interventions, and rebuilt at a later time [44] (cf. Figure 2, B). The development of the two concentration processes of formal inhabitants and inhabitants morphoid is thus not always linked, which contradicts the model.

However, this point of criticism could also offer potential for an adaptation of the model. It may be that the pattern formation in cities cannot be described by an interaction between inhabitants of formal settlements and inhabitants of morphological slums, but by an interaction between inhabitants and green spaces. For example, Fuller and Gaston [46] have shown for European cities that the sum of green spaces in a city correlates with the urban area. There are also increasing attempts to increase the access of residents to green spaces, which can be understood as the need to distribute green spaces regularly throughout the city [47]. In such a model the construction of morphological slums on these green spaces would thus be a secondary process.

As already mentioned, the model [18] describes an idealized theoretical process. In a simulation with spatially homogeneous parameters and initial conditions with clustered concentration peaks (Figure 3), the concentration peaks migrate away from each other over time and form a spatially evenly distributed pattern [21]. Individual concentration peaks split up and move away from each other [22]. Although studies have already shown that morphological slums can also shrink and thus split up [44], it is not usually the case that morphological slums move.

\section{MODEL EXTENSIONS AND PARAMETER IDENTIFICATION}

In the model presented by Pelz et al. [18] the basic possibility of describing complex processes, such as the formation of morphological slums, with simple mathematical models was discussed. Since some discrepancies between the model and empirical results were pointed out in the last sections, we discuss below, on the basis of 
different studies, the possibilities of extending the model according to the empirical findings. Furthermore, with reference to more recent literature, we show how settlement maps could be used to identify underlying mechanisms.

\section{A. Model extension}

In the presented model [18] the stability analysis is carried out with a simple model with spatially and temporally homogeneous parameters. However, as shown above, these assumptions lead to regular arrangements of the objects, which cannot be found in empirical analyses of morphological slums.

In recent studies, however, it is repeatedly stated that the criteria for Turing patterns can be extended in different ways [48]. For example, the interaction could be extended from two to more social groups to address the problem of dichotomous views.

Furthermore, the influence of spatially and temporally heterogeneous media and parameters on pattern formation is intensively discussed [49]. Kozak et al. [50] investigate spatially variable parameters using a Schnakenberg model, probably the best known activator-substrate model. They discuss, similarly to us above, that the strong regularity observed in theoretical or numerical analyses of RD models such as the Schnakenberg or the Gierer-Meinhardt model (one of the first activatorinhibitor models) is often not found in real data. Older works show that jumps in kinetic parameters lead to pattern formation outside the Turing space [51]. On both sides of the discontinuity, the system shows locally restricted pattern formation, even if the parameters are outside the Turing space. The amplitudes become smaller with increasing distance from the discontinuity.

Such locally restricted patterns are not only provoked by parameter jumps, but can also occur in systems with different boundary conditions [52]. Using an analytical method, Benson et al. [53] show that with piecewise constant parameters patterns occur which are restricted to a part of the considered area. However, this behavior is due to the fact that the diffusion coefficient on one side is greater than than the critical diffusion coefficient for a homogeneous area. Furthermore, spatially dependent diffusion coefficients were investigated and it was shown that the wavelengths imprinted on the system from outside can interact with those intrinsically present in the system [54].

The strong topographic and spatially varying social structures in cities of the Global South could therefore be represented in an adapted model by spatially and possibly temporally dependent parameters. Here, the crossdiffusion, which has been neglected up to now, could be included in the model in order to be able to reject the strong assumption of the independent mobilities of the two social groups. The influence of cross-diffusion on the Turing mechanism was also investigated in various works
$[55,56]$.

When discussing these possible model extensions, it must be taken into account that spatially varying parameters introduce further length scales into the system in addition to the characteristic length inherent in the model. Spatial variations of parameters that take geographical influences, such as mountains or rivers, into account should therefore be much greater than the wavelength inherent in the system in order to avoid interference.

\section{B. Parameter identification}

If it is assumed that the formation of morphological slums is based on a mechanism that can be described by partial differential equations, the question of (i) the underlying reaction kinetics and (ii) the corresponding parameters arises. With regard to these questions, enormous progress has been made in recent years. Rudy et al. [57] determined the relevant terms of an equation by determining spatial and temporal derivatives in images and image sequences and were thus able, for example, to deduce underlying dimenson-less parameters by analyzing images. Different groups have further shown how it is possible to determine the parameters of a Turing mechanism from images of contration curves using Bayesian statistics [58, 59].

An application of these and similar methods [60] to time-resolved settlement data could allow an identification of the underlying mechanisms and the related parameters. With these methods it would also be possible to cope with the increasing complexity caused by spatially or temporally varying model parameters, as described in sec. V A.

\section{GENERAL REFLECTIONS}

In sec. V A possible adjustments of the model were presented. Every adaptation of the model bears the risk of overfitting. When adapting the model, it is therefore of utmost importance to emphasize that it cannot be the goal to eliminate every deviation between model and observation. Rather, the aim is to investigate whether adjustments of the model can lead to a broader size distribution and a clustering of the slums in addition to the characteristic length.

In addition to the very specific model discussions presented so far, it must be discussed in general to what extent the model presented by Pelz et al. [18] is suitable for describing the emergence of slums. The model offers the possibility to depict an empirically observed quantity, the similar size of morphological slums, which is apparently independent of the size and the cultural environment of a city, through an interaction of two social groups. In other domains, such as ecology, there are also approaches to explain pattern formation in large-scale 
systems through self-organization [61]. However, there is a discussion about the underlying mechanisms leading to pattern formation and whether or not different processes might interact with each other. They also ask, if a scale-dependent feedback system, like the RD systems described here might be too simple to explain the complex interactions within the systems leading to pattern formation. The approach of a Turing mechanism presented here should therefore be compared in future work with other concepts for pattern formation, e.g. excitable media [62] or positional information [45, 63], in order to determine whether alternatives are available to describe the formation of morphological slums with simple models.

\section{CONCLUSION}

In this paper we investigated the hypothesis of Pelz et al. [18], according to which the formation of slums can be described by a Turing mechanism. This relatively simple model [18] has the advantage over other models [20] of being able to map the characteristic size of slums identified in empirical studies $[13,14]$ by means of a few equations and parameters.

By analysing both, the model and empirical findings on morphological slums, we discussed differences in the spatial relations and development of both patterns. Using spatially and temporally homogeneous parameters, it is possible to map a characteristic size of morphological slums, but this also results in a spatially homogeneous pattern, which contradicts the empirical observations. These differences could be reduced by using parameters that vary in space and time. More critical in the comparison between model and empirical observation is the fact that the temporal development of morphological slums, in contrast to the development of interacting morphogens in Turing patterns, is partially independent of the development of the surrounding structures. One possibility to preserve the model taking this fact into account would be to assume an interaction of open or green spaces and built-up areas, whereby the open spaces could serve as a pre-pattern for the development of morphological slums.

The increasing complexity caused by these or other adaptations can be countered by recent developments in the identification of mechanisms by Bayesian statistics, which allows to detect underlying mechanisms on the basis of settlement information. The main purpose of this work was to examine the limitations of the model [18] in more detail, to address possible points of criticism and to propose possible adjustments. In further studies, the possibility should be considered whether alternative physically motivated concepts exist to model the similar size of morphological slums.

Finally, it should be mentioned that any simplified modelling of human motivation or actions must always reflect and take into account the tension between vividness and adequate appreciation of the reality of life of the individuals described.
[1] U. Habitat, State of the World's Cities 2006/7, New York: United Nations (2006).

[2] M. Kuffer, K. Pfeffer, and R. Sliuzas, Slums from Space-15 Years of Slum Mapping Using Remote Sensing, Remote Sensing 8, 455 (2016).

[3] R. Mahabir, A. Crooks, A. Croitoru, and P. Agouris, The study of slums as social and physical constructs: challenges and emerging research opportunities, Regional Studies, Regional Science 3, 399 (2016).

[4] R. Mahabir, A. Croitoru, A. Crooks, P. Agouris, and A. Stefanidis, A Critical Review of High and Very HighResolution Remote Sensing Approaches for Detecting and Mapping Slums: Trends, Challenges and Emerging Opportunities, Urban Science 2, 8 (2018).

[5] M. Wurm and H. Taubenböck, Detecting social groups from space - Assessment of remote sensing-based mapped morphological slums using income data, Remote Sensing Letters 9, 41 (2018).

[6] A. Gilbert, The Return of the Slum: Does Language Matter?: The return of the slum: does language matter?, International Journal of Urban and Regional Research 31, 697 (2007).

[7] A. Mayne, Slums: the history of a global injustice (Reaktion Books Ltd, London, UK, 2017) oCLC: ocn979568407.

[8] A. Roy, Slumdog Cities: Rethinking Subaltern Urbanism:
Rethinking subaltern urbanism, International Journal of Urban and Regional Research 35, 223 (2011).

[9] J. Wang, M. Kuffer, D. Roy, and K. Pfeffer, Deprivation pockets through the lens of convolutional neural networks, Remote Sensing of Environment 234, 111448 (2019).

[10] M. Kuffer, K. Pfeffer, R. Sliuzas, I. Baud, and M. Maarseveen, Capturing the Diversity of Deprived Areas with Image-Based Features: The Case of Mumbai, Remote Sensing 9, 384 (2017).

[11] D. R. Thomson, M. Kuffer, G. Boo, B. Hati, T. Grippa, H. Elsey, C. Linard, R. Mahabir, C. Kyobutungi, J. Maviti, D. Mwaniki, R. Ndugwa, J. Makau, R. Sliuzas, S. Cheruiyot, K. Nyambuga, N. Mboga, N. W. Kimani, J. P. de Albuquerque, and C. Kabaria, Need for an Integrated Deprived Area "Slum" Mapping System (IDEAMAPS) in Low- and Middle-Income Countries (LMICs), Social Sciences 9, 80 (2020).

[12] H. Taubenböck, N. J. Kraff, and M. Wurm, The morphology of the Arrival City - A global categorization based on literature surveys and remotely sensed data, Applied Geography 92, 150 (2018).

[13] J. Friesen, H. Taubenböck, M. Wurm, and P. F. Pelz, The similar size of slums, Habitat International 73, 79 (2018).

[14] J. Friesen, H. Taubenböck, M. Wurm, and P. F. Pelz, 西

\section{西}


Size distributions of slums across the globe using different data and classification methods, European Journal of Remote Sensing , 1 (2019).

[15] L. Rausch, J. Friesen, L. Altherr, M. Meck, and P. Pelz, A Holistic Concept to Design Optimal Water Supply Infrastructures for Informal Settlements Using Remote Sensing Data, Remote Sensing 10, 216 (2018).

[16] R. Lilford, C. Kyobutungi, R. Ndugwa, J. Sartori, S. I. Watson, R. Sliuzas, M. Kuffer, T. Hofer, J. Porto de Albuquerque, and A. Ezeh, Because space matters: conceptual framework to help distinguish slum from non-slum urban areas, BMJ Global Health 4, e001267 (2019).

[17] J. Friesen, V. Friesen, I. Dietrich, and P. F. Pelz, Slums, Space, and State of Health-A Link between Settlement Morphology and Health Data, International Journal of Environmental Research and Public Health 17, 2022 (2020).

[18] P. F. Pelz, J. Friesen, and J. Hartig, Similar size of slums caused by a Turing instability of migration behavior, Physical Review E 99, 10.1103/PhysRevE.99.022302 (2019).

[19] A. M. Turing, The Chemical Basis of Morphogenesis, Philosophical Transactions of the Royal Society of London. Series B, Biological Sciences 237, 37 (1952).

[20] D. Roy, M. H. Lees, B. Palavalli, K. Pfeffer, and M. A. P. Sloot, The emergence of slums: A contemporary view on simulation models, Environmental Modelling \& Software 59, 76 (2014).

[21] J. D. Murray, Mathematical Biology II - Spatial Models and Biomedical Applications, 3rd ed., Interdisciplinary applied Mathematics, Vol. 18 (Springer-Verlag New York, 2003).

[22] H. Meinhardt, Models of Biological Pattern Formation: From Elementary Steps to the Organization of Embryonic Axes, in Current Topics in Developmental Biology, Vol. 81 (Elsevier, 2008) pp. 1-63.

[23] H. Meinhardt, Turing's theory of morphogenesis of 1952 and the subsequent discovery of the crucial role of local self-enhancement and long-range inhibition, Interface Focus 2, 407 (2012).

[24] D. Kahneman, Thinking, fast and slow (Macmillan, 2011).

[25] Plato, The Republic (Art-type edition. New York : Books, Inc., 1943., 1943).

[26] H. Taubenböck, J. Staab, X. Zhu, C. Geiß, S. Dech, and M. Wurm, Are the Poor Digitally Left Behind? Indications of Urban Divides Based on Remote Sensing and Twitter Data, ISPRS International Journal of GeoInformation 7, 304 (2018).

[27] J. P. Chauvin, E. Glaeser, Y. Ma, and K. Tobio, What is different about urbanization in rich and poor countries? Cities in Brazil, China, India and the United States, Journal of Urban Economics 98, 17 (2017).

[28] M. L. Small, De-Exoticizing Ghetto Poverty: On the Ethics of Representation in Urban Ethnography, City \& Community 14, 352 (2015).

[29] D. Madden, Relative oder absolute poverty lines: a new approach, Review of Income and Wealth 46, 181 (2000).

[30] R. Vigil, Q. Ouyang, and H. L. Swinney, Turing patterns in a simple gel reactor, Physica A: Statistical Mechanics and its Applications 188, 17 (1992).

[31] H. Rosling, O. Rosling, and A. R. Rönnlund, Factfulness: ten reasons we're wrong about the world - and why things are better than you think (2019) oCLC: 1128839313.
[32] N. Levashova, A. Sidorova, A. Semina, and M. Ni, A Spatio-Temporal Autowave Model of Shanghai Territory Development, Sustainability 11, 3658 (2019).

[33] S. M. Low, The edge and the center: Gated communities and the discourse of urban fear, American anthropologist 103, 45 (2001).

[34] E. A. Codling, M. J. Plank, and S. Benhamou, Random walk models in biology, Journal of The Royal Society Interface 5, 813 (2008).

[35] M. C. González, C. A. Hidalgo, and A.-L. Barabási, Understanding individual human mobility patterns, Nature 453, 779 (2008).

[36] C. Castellano, S. Fortunato, and V. Loreto, Statistical physics of social dynamics, Reviews of Modern Physics 81, 591 (2009).

[37] C. Song, Z. Qu, N. Blumm, and A.-L. Barabasi, Limits of Predictability in Human Mobility, Science 327, 1018 (2010).

[38] V. Kulkarni, A. Mahalunkar, B. Garbinato, and J. Kelleher, Examining the Limits of Predictability of Human Mobility, Entropy 21, 432 (2019).

[39] M. Kuffer, F. Orina, R. Sliuzas, and H. Taubenböck, Spatial patterns of slums: Comparing African and Asian cities (IEEE, 2017) pp. 1-4.

[40] J. Hartig, J. Friesen, and P. F. Pelz, Spatial relations of slums: size of slum clusters, in 2019 Joint Urban Remote Sensing Event (JURSE) (IEEE, Vannes, France, 2019) pp. 1-4.

[41] J. Schnakenberg, Simple chemical reaction systems with limit cycle behaviour, Journal of Theoretical Biology 81, 389 (1979).

[42] O. Gruebner, J. Sachs, A. Nockert, M. Frings, M. M. H. Khan, T. Lakes, and P. Hostert, Mapping the Slums of Dhaka from 2006 to 2010, Dataset Papers in Science 2014, 1 (2014).

[43] S. Kondo and T. Miura, Reaction-Diffusion Model as a Framework for Understanding Biological Pattern Formation, Science 329, 1616 (2010).

[44] R. Liu, M. Kuffer, and C. Persello, The Temporal Dynamics of Slums Employing a CNN-Based Change Detection Approach, Remote Sensing 11, 2844 (2019).

[45] J. B. A. Green and J. Sharpe, Positional information and reaction-diffusion: two big ideas in developmental biology combine, Development 142, 1203 (2015).

[46] R. A. Fuller and K. J. Gaston, The scaling of green space coverage in European cities, Biology Letters 5, 352 (2009).

[47] T. Byomkesh, N. Nakagoshi, and A. M. Dewan, Urbanization and green space dynamics in Greater Dhaka, Bangladesh, Landscape and Ecological Engineering 8, 45 (2012).

[48] M. Kuznetsov and A. Polezhaev, Widening the criteria for emergence of Turing patterns, Chaos: An Interdisciplinary Journal of Nonlinear Science 30, 033106 (2020).

[49] A. L. Krause, V. Klika, T. E. Woolley, and E. A. Gaffney, From one pattern into another: analysis of Turing patterns in heterogeneous domains via WKBJ, Journal of The Royal Society Interface 17, 20190621 (2020).

[50] M. Kozák, E. A. Gaffney, and V. Klika, Pattern formation in reaction-diffusion systems with piecewise kinetic modulation: An example study of heterogeneous kinetics, Physical Review E 100, 10.1103/PhysRevE.100.042220 (2019).

[51] K. Page, P. K. Maini, and N. A. Monk, Pattern forma- 
tion in spatially heterogeneous Turing reaction-diffusion models, Physica D: Nonlinear Phenomena 181, 80 (2003).

[52] P. Maini and M. Myerscough, Boundary-driven instability, Applied Mathematics Letters 10, 1 (1997).

[53] D. Benson, J. Sherratt, and P. Maini, Diffusion driven instability in an inhomogeneous domain, Bulletin of Mathematical Biology 55, 365 (1993).

[54] J.-P. Voroney, A. Lawniczak, and R. Kapral, Turing pattern formation in heterogenous media, Physica D: Nonlinear Phenomena 99, 303 (1996).

[55] V. K. Vanag and I. R. Epstein, Cross-diffusion and pattern formation in reaction-diffusion systems, Phys. Chem. Chem. Phys. 11, 897 (2009).

[56] G. Gambino, M. Lombardo, and M. Sammartino, Pattern formation driven by cross-diffusion in a 2D domain, Nonlinear Analysis: Real World Applications 14, 1755 (2013).

[57] S. H. Rudy, S. L. Brunton, J. L. Proctor, and J. N. Kutz, Data-driven discovery of partial differential equations, Science Advances 3, e1602614 (2017).
[58] E. Campillo-Funollet, C. Venkataraman, and A. Madzvamuse, Bayesian Parameter Identification for Turing Systems on Stationary and Evolving Domains, Bulletin of Mathematical Biology 81, 81 (2019).

[59] A. Kazarnikov and H. Haario, Statistical approach for parameter identification by Turing patterns, Journal of Theoretical Biology 501, 110319 (2020).

[60] H. Zhao, B. D. Storey, R. D. Braatz, and M. Z. Bazant, Learning the Physics of Pattern Formation from Images, Physical Review Letters 124, 10.1103/PhysRevLett.124.060201 (2020).

[61] R. M. Pringle and C. E. Tarnita, Spatial SelfOrganization of Ecosystems: Integrating Multiple Mechanisms of Regular-Pattern Formation, Annual Review of Entomology 62, 359 (2017).

[62] E. Meron, Pattern formation in excitable media, Physics Reports 218, 1 (1992).

[63] I. Salazar-Ciudad, Mechanisms of pattern formation in development and evolution, Development 130, 2027 (2003). 
A TURING MODEL SIMULATION OF AN ACTIVATOR-SUBSTRATE MODEL

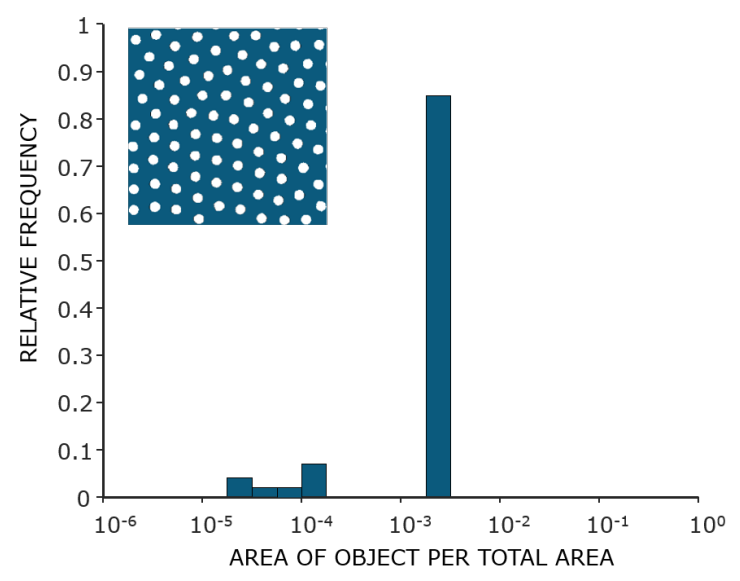

B MORPHOLOGICAL SLUMS

EMPIRICAL DATA OF DHAKA (2010)

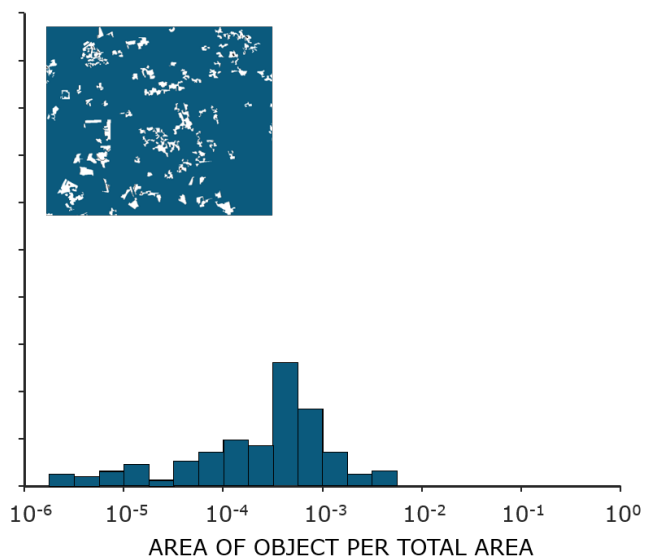

FIG. 1. A Resulting pattern of a simulation of a common activator-substrate model with Schnakenberg type kinetics [41] with homogeneous parameters and corresponding size distribution. The binary representation was created by coloring all locations where the concentration of the activator exceeded a threshold white and all other areas blue. The small objects at the edge of the simulation area lead to the outliers between $10^{-5}$ and $10^{-4}$. B Empirical pattern of morphological slums in a small area $\left(3 \times 2.5 \mathrm{~km}^{2}\right)$ of Dhaka (2010), collected by [42] and corrosponding size distribution.

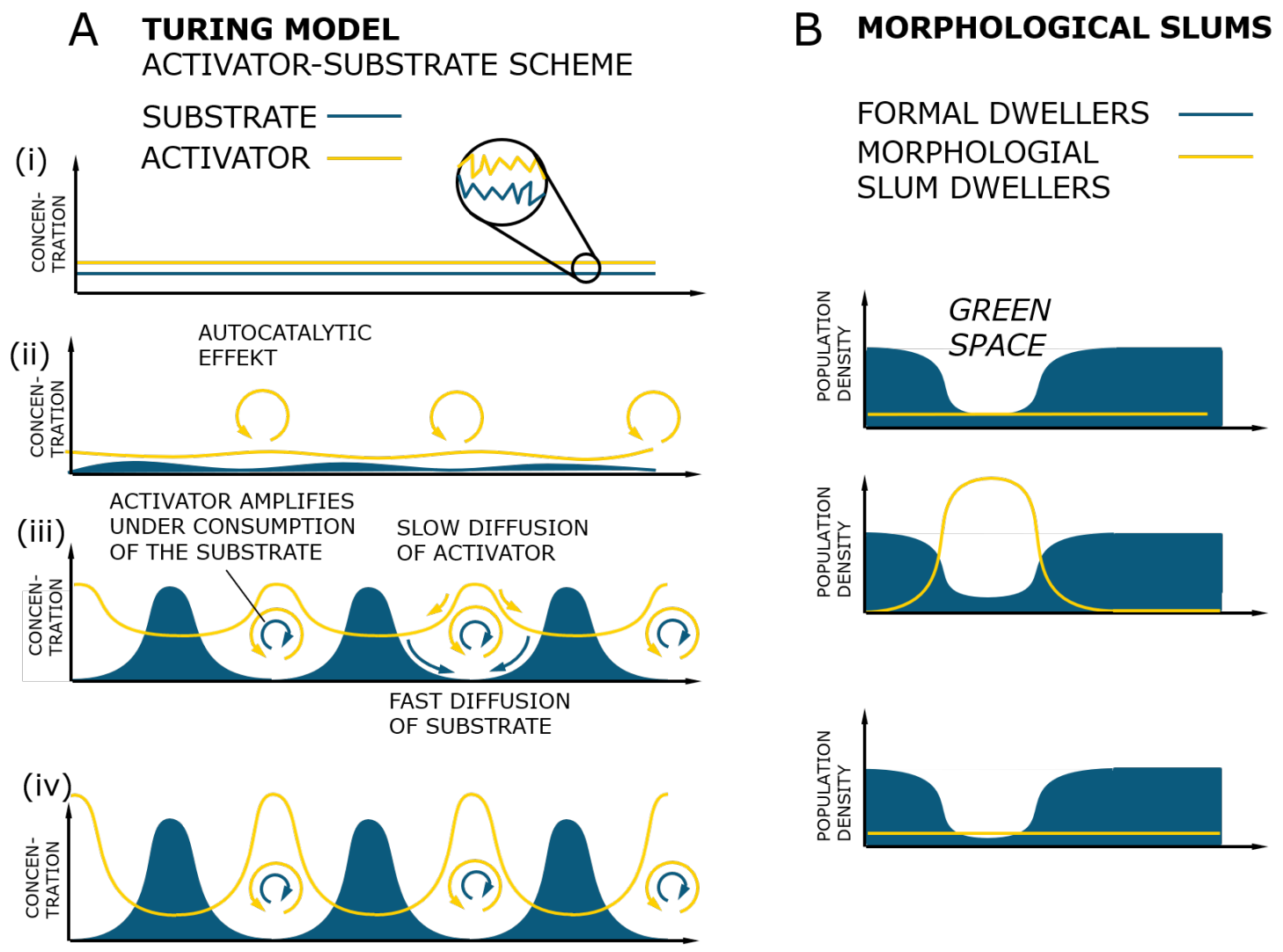

FIG. 2. A Representation of a self-reinforcing effect in an activator-substrate model. The illustration on the left side is inspired by Green and Sharpe [45] and Meinhardt [22, 23]. B Schematic representation of the temporal development and dissolution of a morphological slum on a green space. 
(i)

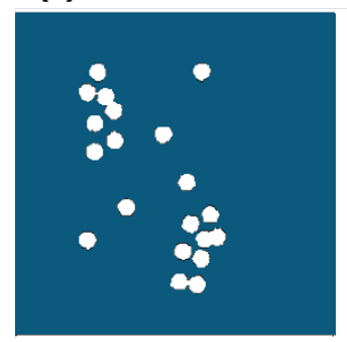

(ii)

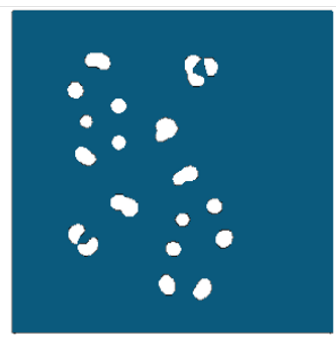

(iii)

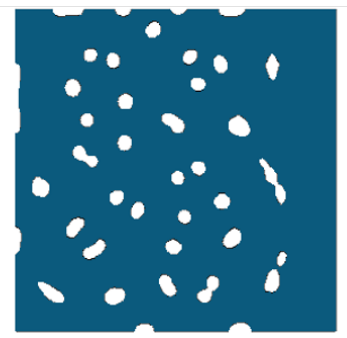

(iv)

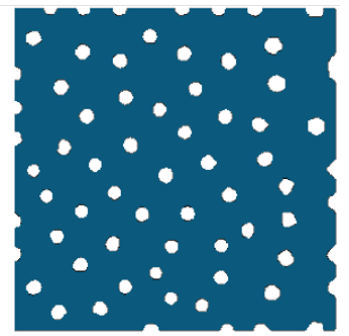

FIG. 3. Qualitative illustration of a activator-substrate model with clusters as initial condition and homogeneous parameters. Initial condition (i) $t=0$, (ii) $t=0.16 t_{\text {end }}$, (iii) $t=0.27 t_{\text {end }}$ and end (iv) $t=t_{\text {end }} . t_{\text {end }}$ is defined as the time at which the concentration peaks no longer move spatially. 